\title{
A Note on an Approximation in the Theory of Random Walk*
}

\author{
By S. Syōno \\ Geophysical Institute, Tokyo University \\ (Manuscript received 10 September 1966)
}

As is well known in the theory of random walk, a probability function is given by an intergral form which includes Bessel functions.

Above all, the theory in two-dimensional case is often used. For instance, Syōno and Tanaka (1966) showed that the frequency distributions of wind speed and direction in turbulent flow can be calculated by use of random walk theory in two-dimension.

Now, after Kluyver's formula (see Watson, 1922 ), the probability function is expressed as,

$$
\begin{array}{cc}
P_{N}\left(r \mid a_{1}, a_{2} \cdots \cdots a_{N}\right)= & r \int_{0}^{\infty} J_{1}(r t) \\
\times \prod_{m=1}^{N} J_{0}(a m t) d t & \left(r \leqq \sum_{m=1}^{N} a_{m}\right) \\
& \left(r>\sum_{m=1}^{N} a_{m}\right)
\end{array}
$$

where $P_{N}(r)$ denotes the probability that the final distance is less than $r$, and $a_{1}, a_{2} \cdots \cdots a_{N}$ denote the length of each step.

Hereafter, for the sake of simplicity, we assume that the length of each step is equal unity, i.e.,

$$
a_{1}=a_{2}=\cdots \cdots \cdot a_{N}=1
$$

By differentiating the equation (1) with respect to $r$, we obtain

$$
B_{N}(r)= \begin{cases}\frac{1}{2 \pi} \int_{0}^{\infty} t J_{0}(r t)\left\{J_{0}(t)\right\}^{N} & (r \leqq N) \\ 0 & (r>N)\end{cases}
$$

where $B_{N}(r)$ denotes the probability that the final distance is confined in $r$ and $r+d r$.

Since the functional form of $B_{N}(r)$ is too complicated to be calculated analytically, this probability function $B_{N}(r)$ is often approxi-

* Division of Meteorology, Contribution No. 151. mated by Gaussian distribution as

$$
B_{N}(r) \approx G_{N}(r)=\frac{2}{\sqrt{N \pi}} \exp \cdot\left(-\frac{r^{2}}{N}\right)
$$

The quantitative discussion about the validity of this approximation has, however, not yet been done, except only the fact that $B_{N}(r)$ tends to $G_{N}(r)$ when $N$ becomes large.

In this short note, numerical calculations of $B_{N}(r)$ and $G_{N}(r)$ are carried out in order to examine the validity of approximation by Gaussian distribution. The results of computations are shown in the figures. In the case of $N=1$ ( $\delta$-function) and $N 2$, the approximation has so less meaning that they are omitted from the figures.

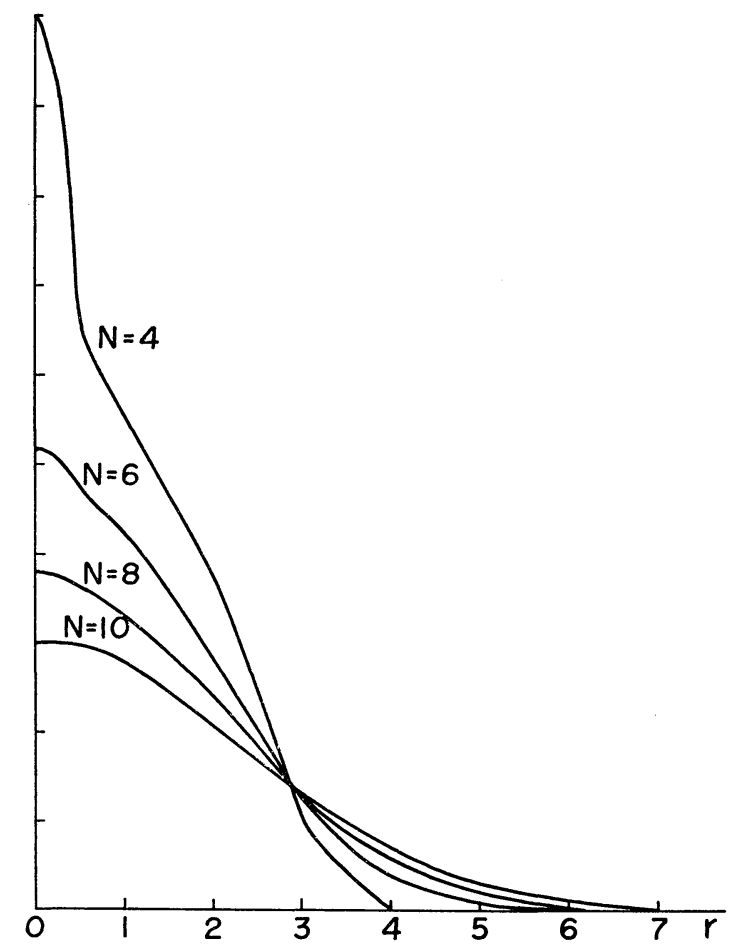

Fig. 1. Computed values of $B_{N}(r)$. 


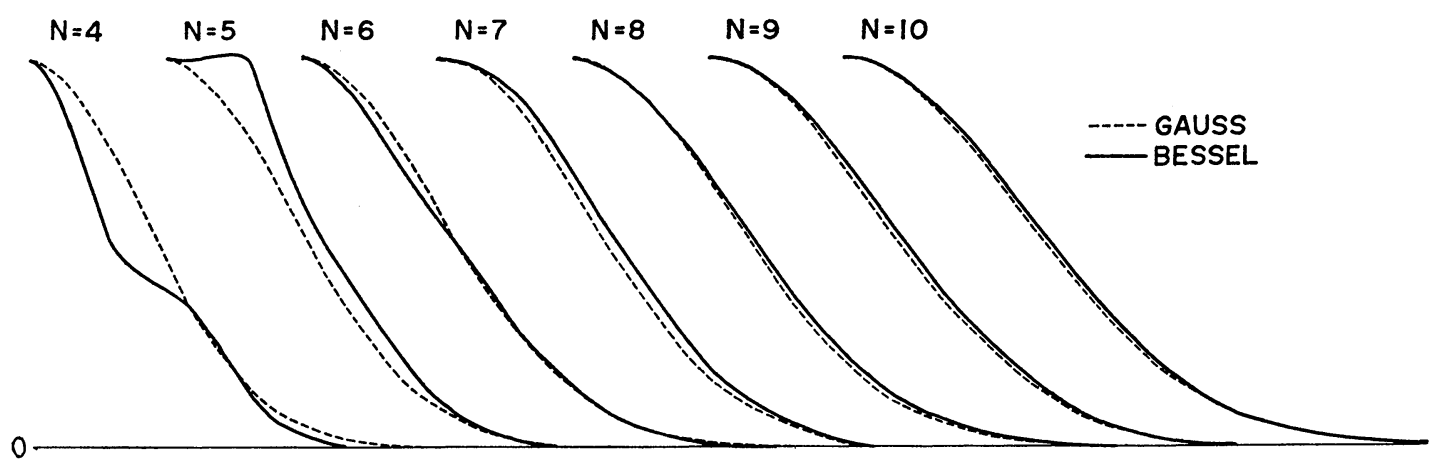

Fig. 2. Comparison between $B_{N}(r)$ and $G_{N}(r)$.

Each function is not normalized but arranged to be unity at $r=0$.

It can be easily concluded from these is about $5 \%$ and becomes about $1 \%$ when results that superposition of six waves or $N=10$, as is shown in Fig. 3. more, whose phase-angles are at random, makes its probability of existence to be near Gaussian. When $N$ is 6 , the probable error

Finally it should be remarked that the radial distribution of $B_{N}(r)$ has a discontinuity at $r=N$, as is seen in eq. (3). In numerical

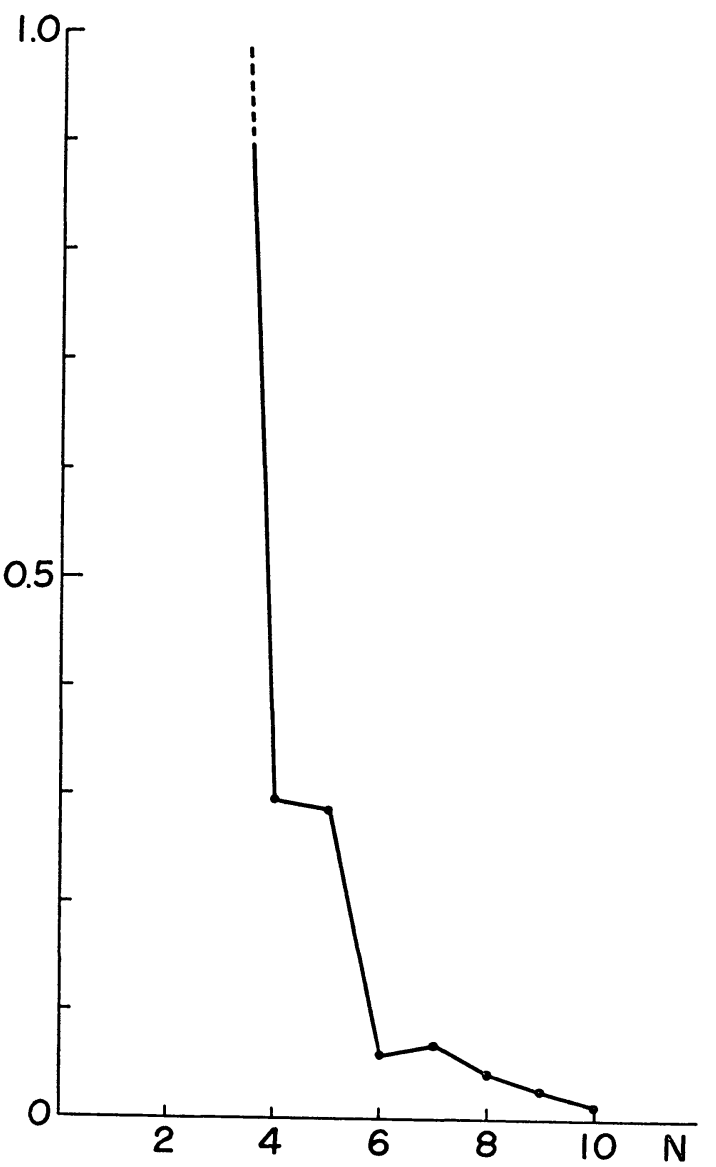

Fig. 3 a. Convergence of $B_{N}$ to $G_{N}$ with respect to $N$.

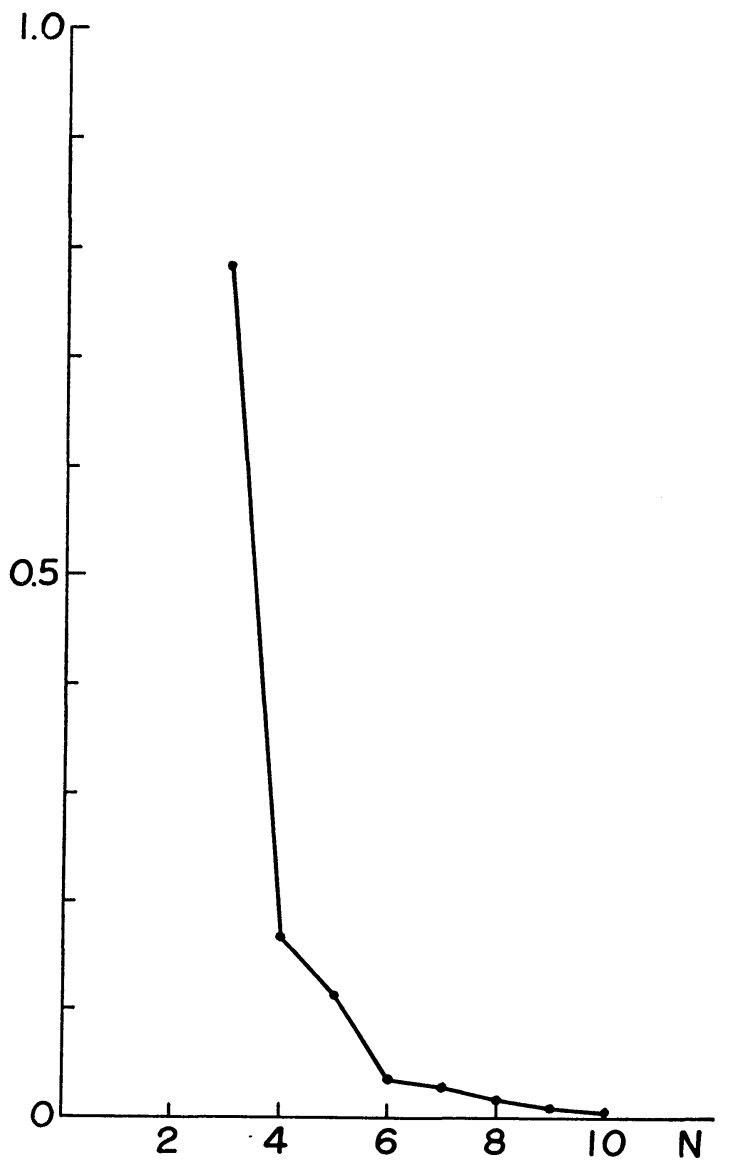

Fig. 3 b. Same as Fig. 3 a. 
computations, however, actual values of $B_{N}(r)$ are so small that they can be regarded as continuous functions, except for the case of small $N$.

These computations were carried out by N. Nakamura and later revised by. I. Hirota of Tokyo University.

\section{References}

Syōno, S. and H. Tanaka, 1966: On the frequency distributions of wind speed and direction in turbulent flow. J. meteor. Soc. Japan, 44, 89100.

Watson, G. N., 1922: Theory of Bessel functions. Cambridge Univ. Press.

\section{Random walk の理論における一つの近似について}

$$
\text { 正野重方 }
$$

東京大学理学部地球物理学教室 